\title{
Part-products of 1-free integer compositions
}

\author{
Caroline Shapcott* \\ Department of Mathematics \\ Drexel University \\ Philadelphia, PA 19104, U.S.A. \\ cshapcott@drexel.edu
}

Submitted: Jun 24, 2011; Accepted: Sep 23, 2011; Published: Dec 19, 2011

Mathematics Subject Classifications: 05A16, 60C05, 60F05, 60G40

\begin{abstract}
If $\vec{\lambda}$ is a composition of the positive integer $n$, define $\mathbf{B}(\vec{\lambda})$ to be the product of the parts of $\vec{\lambda}$. We present a modified version of Hitczenko's stopped sequence construction that leads to a proof of the asymptotic lognormality of $\mathbf{B}$ for random 1-free compositions (compositions containing no parts of size 1).
\end{abstract}

\section{Introduction}

A composition of $n$ is a sequence of positive integers whose terms, referred to as parts, sum to $n$. In recent years, there has been considerable interest in compositions with restrictions on the sizes of the parts. One set of restricted compositions that has been studied extensively is that of Carlitz compositions, whose adjacent part sizes cannot be equal $[8,13,15]$. A generalization of Carlitz compositions is introduced by Bender and Canfield in $[2,3,4]$ where the authors study locally restricted compositions, whose part sizes are restricted to a certain set depending on the position of the part. A variety of other restricted compositions have been studied, including complete and gap-free compositions, whose part sizes form an interval [10]; compositions with distinct part sizes [14]; compositions with the largest part in the first position [16]; compositions with no parts of size $2[6]$; and compositions whose parts sizes are restricted to a general set $[1,5]$.

Problems that are straightforward for unrestricted compositions can become quite complicated when such restrictions are imposed. In [18] it is proven that, for unrestricted compositions, the product of parts is asymptotically lognormal. The proof relies heavily on a construction, devised by Hitczenko and others, that models compositions with

*Supported in part by N.S.A. grant H98230-09-1-0062. Portions of this work will appear in the author's doctoral dissertation, written under the direction of Eric Schmutz at Drexel University. 
stopped sequences of geometric random variables. However, the proof does not extend immediately to any restricted part-size setting of which we are aware. In particular, one might expect the proof to extend to compositions with no parts of size 1, since those parts do not contribute to the product. However, non-trivial adaptations are needed to make Hitczenko's technique usable in this setting. The main result in this manuscript is Theorem 1.

Theorem 1. Let $P_{n}$ be the uniform probability measure on the set of 1-free compositions of $n$, and let $\phi=\frac{1+\sqrt{5}}{2}$ be the golden ratio. Define the constants $\mu=\sum_{k=2}^{\infty} \frac{\log k}{\phi^{k}}, \nu=\sum_{k=2}^{\infty} \frac{k \log k}{\phi^{k}}$, $\bar{\mu}=\sum_{k=2}^{\infty} \frac{(\log k)^{2}}{\phi^{k}}$, and $\gamma^{2}=\left(1+\frac{\phi}{5}\right) \mu^{2}-\left(\frac{2}{1+\phi^{2}}\right) \mu \nu+\bar{\mu}$. Then

$$
P_{n}\left(\frac{\log \mathbf{B}-\frac{n \mu}{1+\phi^{2}}}{\sqrt{\frac{n \gamma^{2}}{1+\phi^{2}}}} \leq x\right)=\frac{1}{\sqrt{2 \pi}} \int_{-\infty}^{x} e^{-t^{2} / 2} d t+O\left(\left(\frac{\log n}{\sqrt{n}}\right)^{1 / 3}\right)
$$

The rate of convergence is uniform for $|x| \leq\left(\frac{\sqrt{n}}{\log n}\right)^{2 / 3}$.

We present the new construction used for modeling 1-free compositions, as well as some preliminary results, before presenting the proof of the main theorem. Throughout this manuscript, we denote the set of 1-free compositions of $n$ as $\Lambda_{n}$ and an individual composition having $\kappa_{n}$ parts as $\vec{\lambda}=\left(\lambda_{1}, \ldots, \lambda_{\kappa_{n}}\right)$. $P_{n}$ denotes the uniform probability measure on $\Lambda_{n}$, and $E_{n}$ denotes the expected value with respect to $P_{n}$. If $F$ is a formal power series in $x$, then we write $\left[x^{n}\right] F$ to denote the coefficient of $x^{n}$ in $F$.

\section{Expected value of $B$ and $\log B$}

Moments of $\mathbf{B}$ can be computed using the following lemma. Let $\Lambda_{n}^{S}$ denote the set of all compositions of $n$ with parts from some set of positive integers $S$, and let $\Lambda_{n, d}^{S}$ denote the set of all such compositions having $d$ parts.

Lemma 1. For $a \in \mathbb{C}$, define $\alpha_{n}=\sum_{\vec{\lambda} \in \Lambda_{n}^{S}} \mathbf{B}(\vec{\lambda})^{a}$. The generating function for the sequence $\alpha_{n}$ is

$$
\sum_{n} \alpha_{n} x^{n}=\frac{1}{1-\sum_{k \in S} k^{a} x^{k}}
$$

Proof. Let $G_{a}(x)=\sum_{k \in S} k^{a} x^{k}$. Then the coefficient of $x^{n}$ in $G_{a}(x)^{d}$ is the sum of the $a$-th power of the part-products over all compositions with $d$ parts:

$$
\left[x^{n}\right] G_{a}(x)^{d}=\left[x^{n}\right] \sum_{\vec{\lambda} \in \Lambda_{n, d}^{S}} \prod_{i} \lambda_{i}^{a} x^{\lambda_{i}}=\left[x^{n}\right] \sum_{\vec{\lambda} \in \Lambda_{n, d}^{S}} \mathbf{B}(\vec{\lambda})^{a} x^{n} .
$$


Summing on $d$ we get

$$
\alpha_{n}=\left[x^{n}\right] \sum_{d=1}^{n} G_{a}(x)^{d}=\left[x^{n}\right] \frac{1}{1-G_{a}(x)} .
$$

Lemma 1 is a new generalization of the following widely known generating function for the number of compositions with parts from a general set [7].

Corollary 1. The generating function for the number of compositions of $n$ with parts from the set $S$ is

$$
\sum_{n}\left|\Lambda_{n}^{S}\right| x^{n}=\frac{1}{1-\sum_{k \in S} x^{k}}
$$

Corollary 2. If $\vec{\lambda}$ is chosen from a uniform distribution on the set of compositions of $n$ into parts from the set $S$, then the expected value of the random variable $\mathbf{B}$ is

$$
\left[x^{n}\right] \frac{1}{1-\sum_{k \in S} k x^{k}} /\left[x^{n}\right] \frac{1}{1-\sum_{k \in S} x^{k}} .
$$

In [18], a probabilistic proof is given to show that the average product over all unrestricted compositions of $n$ is $F_{2 n} / 2^{n-1}$, where $F_{2 n}$ is the $2 n$-th Fibonacci number. An alternative approach is to cite Corollary 2 with $S=\mathbb{Z}_{+}$. It is interesting that the Fibonacci numbers also appear in two special cases that are important for this manuscript. The most prominent occurrence is in the probabilistic construction that will be presented in the next section. In addition, Corollary 1 with $S=\{k: k \geq 2\}$ gives the following known result.

Corollary 3. The number of 1-free compositions of $n$ is $F_{n-1}$.

Corollary 2 also yields an asymptotic formula for the expected value of $\mathbf{B}$ for 1-free compositions.

Theorem 2. Let $\rho_{1}, \rho_{2}$, and $\rho_{3}$ be the distinct real roots of the function $x^{3}-x^{2}-2 x+1$. If $\vec{\lambda}$ is chosen from a uniform distribution on the set of 1-free compositions of $n$, the expected value of the product of parts is

$$
E_{n}(\mathbf{B})=\frac{1}{F_{n-1}} \sum_{i=1}^{3} \frac{\left(1-\rho_{i}\right)^{2} \rho_{i}^{-n}}{2 \rho_{i}+2 \rho_{i}^{2}-3 \rho_{i}^{3}} .
$$

Asymptotically, the expected value is

$$
E_{n}(\mathbf{B}) \sim \frac{\sqrt{5}}{\phi^{n-1}} \cdot \frac{\left(1-\rho_{1}\right)^{2} \rho_{1}^{-n}}{2 \rho_{1}+2 \rho_{1}^{2}-3 \rho_{1}^{3}}=(1.09 \ldots)(1.3887 \ldots)^{n} .
$$

As a result of Theorem 1, we know that the expected value of the log product of parts is approximately $\frac{n \mu}{1+\phi^{2}}$. This agrees with results from [18] where the following precise estimate is obtained. 
Theorem 3. If $\vec{\lambda}$ is chosen from a uniform distribution on the set of 1-free compositions of $n$, the expected value of the log product of parts is

$$
E_{n}(\log \mathbf{B})=\frac{n \mu}{1+\phi^{2}}+\left(\frac{\phi}{5}+1\right) \mu-\frac{\nu}{1+\phi^{2}}+O\left(\frac{n \log n}{\phi^{n}}\right)
$$

The proof of Theorem 3 requires the probabilistic construction presented in Section 3 as well as a detailed consideration of the distribution of the last part $\lambda_{\kappa_{n}}$. The proof is omitted because it involves lengthy calculations similar to those in the proof of the asymptotic lognormality of $\mathbf{B}$.

\section{Stopped sequence construction}

We begin the new construction by defining an auxiliary space $\Lambda_{n}^{*}$ that contains all 1free compositions of $n$ as well as all compositions of $n$ that are 1-free except for the last part. Let $\Omega_{n}$ be the set of all $n$-length sequences $\vec{\omega}=\left(\omega_{1}, \ldots, \omega_{n}\right)$ with $\omega_{i} \geq 2$, and let $\tau_{n}=\min \left\{t \geq 1: \omega_{1} \cdots+\omega_{t} \geq n\right\}$ be a stopping time associated with the sequence $\vec{\omega}$. We can then construct random compositions of $n$ by defining the function

$$
H_{n}(\vec{\omega})=\left(\omega_{1}, \cdots, \omega_{\tau_{n}-1}, n-\sum_{i=1}^{\tau_{n}-1} \omega_{i}\right)
$$

Notice that $H_{n}$ does not always return 1-free compositions of $n$, since it is possible for the last part to be a 1 . Moreover, if we define a probability measure on the space $\Lambda_{n}^{*}$ in the manner of $[10,11,12]$, then $H_{n}$ does not select compositions uniformly.

Formally, if we select the $\omega_{i}$ 's independently with probability $Q_{n}\left(\omega_{i}=k\right)=\frac{1}{\phi^{k}}$ for $k \geq 2$ and define a probability measure on $\Omega_{n}$ by

$$
Q_{n}(\vec{\omega})=\frac{1}{\phi^{\omega_{1}+\cdots+\omega_{n}}}
$$

then we can define a probability measure $Q_{n} \circ H_{n}^{-1}$ on $\Lambda_{n}^{*}$, where $H_{n}^{-1}$ is described as follows. For a composition $\vec{\lambda}$ with $\kappa_{n}=k$ parts and last part $j$,

$$
H_{n}^{-1}(\vec{\lambda})= \begin{cases}\left\{\vec{\omega} \in \Omega_{n}: \omega_{i}=\lambda_{i}, i=1, \ldots, k-1\right\} & \text { if } j=1 \\ \left\{\vec{\omega} \in \Omega_{n}: \omega_{i}=\lambda_{i}, i=1, \ldots, k-1 \text { and } \omega_{k} \geq 2\right\} & \text { if } j>1 .\end{cases}
$$

Although $Q_{n} \circ H_{n}^{-1}$ is not uniform, it is conditionally uniform given the size of the last part:

$$
Q_{n}\left(H_{n}^{-1}(\vec{\lambda})\right)= \begin{cases}\prod_{i=1}^{k-1} \frac{1}{\phi^{\lambda_{i}}}=\frac{1}{\phi^{n-1}} & \text { if } j=1 \\ \prod_{i=1}^{k-1} \frac{1}{\phi^{\lambda_{i}}} \sum_{i=j}^{\infty} \frac{1}{\phi^{i}}=\frac{1}{\phi^{n-2}} & \text { if } j>1 .\end{cases}
$$


For ease of use, we let $P_{n}^{*}=Q_{n} \circ H_{n}^{-1}$ and denote the expected value with respect to $P_{n}^{*}$ as $E_{n}^{*}$. We denote the expected value with respect to $Q_{n}$ as $E_{n}^{Q}$.

The following observation will complete the new construction so that it is useful for our analysis of the product of parts. If $X^{*}$ is any random variable on $\Lambda_{n}^{*}$, then there is a corresponding random variable $X$ on $\Omega_{n}$ defined in the obvious way, $X(\vec{\omega})=X^{*}\left(H_{n}(\vec{\omega})\right)$, and having the same distribution as $X^{*}$ :

Lemma 2. If $X^{*}$ is any random variable on $\Lambda_{n}^{*}$ and if $X=X^{*} \circ H_{n}$, then for all real numbers $y$,

$$
P_{n}^{*}\left(X^{*}=y\right)=Q_{n}(X=y)
$$

\section{Number of parts}

For random unrestricted compositions, the number of parts is a binomial random variable that has an expected value of $\frac{n+1}{2}$. A local limit theorem is proved in [1] for the number of parts of a composition with parts from a general set, but more specific results for 1-free compositions are needed for the proof of Theorem 1.

Theorem 4. If $\vec{\lambda}$ is chosen from a uniform distribution on the set of 1-free compositions of $n$, the expected number of parts is

$$
E_{n}\left(\kappa_{n}\right)=\frac{n}{1+\phi^{2}}+\frac{\phi}{5}+O\left(\frac{n}{\phi^{2 n}}\right) .
$$

Proof. Observe that the total number of parts over all compositions in $\Lambda_{n}^{S}$ is

$$
\left|\Lambda_{n}^{S}\right| E_{n}\left(\kappa_{n}\right)=\sum_{k=1}^{n} k\left|\Lambda_{n, k}^{S}\right|=\sum_{k=1}^{n} \sum_{d=k}^{n}\left|\Lambda_{n, d}^{S}\right| .
$$

Using the fact that the coefficient of $x^{n}$ in $G_{0}(x)^{d}$ is equal to $\left|\Lambda_{n, d}^{S}\right|$, as in the proof of Lemma 1, we have

$$
\sum_{k=1}^{n} \sum_{d=k}^{n}\left|\Lambda_{n, d}^{S}\right|=\left[x^{n}\right] \sum_{k=1}^{n} \sum_{d=k}^{n} G_{0}(x)^{d}=\left[x^{n}\right] \frac{G_{0}(x)}{\left(1-G_{0}(x)\right)^{2}} .
$$

Letting $S=\{k: k \geq 2\}$, so that $G_{0}(x)=\sum_{k=2}^{\infty} x^{k}$, and combining (2) with Corollary 3 , we obtain

$$
\begin{aligned}
E_{n}\left(\kappa_{n}\right)= & \frac{1}{F_{n-1}}\left[x^{n}\right] \frac{G_{0}(x)}{\left(1-G_{0}(x)\right)^{2}} \\
& =\frac{1}{F_{n-1}}\left(n\left(\frac{3}{5} F_{n-1}-\frac{1}{5} F_{n}\right)+\frac{F_{n}}{5}\right) \\
& =\frac{n}{1+\phi^{2}}+\frac{\phi}{5}+O\left(\frac{n}{\phi^{2 n}}\right) .
\end{aligned}
$$


A pertinent part of the lognormality proof is to show that the number of parts of a composition is concentrated around its mean. For unrestricted compositions, Chernoff bounds can be applied directly to obtain an exponentially small upper bound. For 1-free compositions, we present the following result.

Lemma 3. There exists a constant $C_{1}>0$ such that

$$
Q_{n}\left(\left|\tau_{n}-\frac{n}{1+\phi^{2}}\right|>\sqrt{n} \log n\right) \leq \frac{C_{1}}{n^{1+\phi^{2}}}
$$

Proof. For positive integers $\alpha$, define $S_{\alpha}=\sum_{i=1}^{\alpha} \omega_{i}$. Then for $t>0$,

$$
Q_{n}\left(\tau_{n} \leq \alpha\right)=Q_{n}\left(S_{\alpha} \geq n\right)=Q_{n}\left(e^{t S_{\alpha}} \geq e^{t n}\right)
$$

By Markov's inequality and the independence of the $\omega_{i}$ 's,

$$
Q_{n}\left(e^{t S_{\alpha}} \geq e^{t n}\right) \leq \frac{E_{n}^{Q}\left(e^{t S_{\alpha}}\right)}{e^{t n}}=\frac{E_{n}^{Q}\left(e^{t \omega_{1}}\right)^{\alpha}}{e^{t n}}
$$

We compute the moment generating function of $\omega_{1}$ to get, for $0<t<\log \phi$,

$$
E_{n}^{Q}\left(e^{t \omega_{1}}\right)=\sum_{k=2}^{\infty} e^{t k} Q_{n}\left(\omega_{1}=k\right)=\sum_{k=2}^{\infty}\left(\frac{e^{t}}{\phi}\right)^{k}=\frac{\left(\frac{e^{t}}{\phi}\right)^{2}}{1-\frac{e^{t}}{\phi}}=\frac{e^{2 t}}{1-\frac{e^{t}-1}{\phi-1}} .
$$

If we let $u=\frac{e^{t}-1}{\phi-1}$, then

$$
Q_{n}\left(\tau_{n} \leq \alpha\right) \leq \frac{\left(\frac{e^{2 t}}{1-u}\right)^{\alpha}}{e^{t n}}=\frac{e^{2 t \alpha}}{e^{\alpha \log (1-u)} e^{t n}}=e^{-t n+2 t \alpha-\alpha \log (1-u)} .
$$

Now it remains to estimate the exponent in the right-hand side of (3). Define $\alpha=$ $\left\lfloor\frac{n}{1+\phi^{2}}-\sqrt{n} \log n\right\rfloor$ and let $t=\frac{1}{\sqrt{n}}$. Then, with an application of the Taylor expansion $\log (1-u)=-\frac{1}{\phi-1}\left(\frac{1}{\sqrt{n}}+\frac{1}{2 n}\right)-\frac{1}{2 n(\phi-1)^{2}}+O\left(\frac{1}{n^{3 / 2}}\right)$, we have

$$
\begin{aligned}
-t n & =-\sqrt{n}, \\
2 t \alpha & =\frac{2 \sqrt{n}}{1+\phi^{2}}-2 \log n+O\left(\frac{1}{\sqrt{n}}\right), \\
-\alpha \log (1-u) & =\frac{\sqrt{n}}{\left(1+\phi^{2}\right)(\phi-1)}-\frac{\log n}{\phi-1}+O(1) .
\end{aligned}
$$

Now (4), (5), and (6) can be used to make substitutions in the exponent of (3). When like terms are combined, the coefficient of $\sqrt{n}$ is

$$
-1+\frac{2}{1+\phi^{2}}+\frac{1}{\left(1+\phi^{2}\right)(\phi-1)}=0
$$


and the coefficient of $\log n$ is

$$
-2-\frac{1}{\phi-1}=-\left(1+\phi^{2}\right)
$$

Since the remaining terms are $O(1)$, we have for some $C_{1}>0$,

$$
Q_{n}\left(\tau_{n} \leq \frac{n}{1+\phi^{2}}-\sqrt{n} \log n\right) \leq e^{-\left(1+\phi^{2}\right) \log n+O(1)} \leq \frac{C_{1}}{n^{1+\phi^{2}}}
$$

A similar calculation using $\beta=\left\lceil\frac{n}{1+\phi^{2}}+\sqrt{n} \log n\right\rceil$ gives the inequality in the other direction.

Corollary 4. There exists a constant $C_{2}>0$ such that

$$
P_{n}\left(\left|\kappa_{n}-\frac{n}{1+\phi^{2}}\right|>\sqrt{n} \log n\right) \leq \frac{C_{2}}{n^{1+\phi^{2}}} .
$$

Proof. By Lemma 2, $P_{n}^{*}\left(\kappa_{n} \geq j\right)=Q_{n}\left(\tau_{n} \geq j\right)$. Thus the inequality in Lemma 3 remains unchanged for $P_{n}^{*}$. Let $A$ be the event $\left|\kappa_{n}-\frac{n}{1+\phi^{2}}\right| \geq \sqrt{n} \log n$. Then

$$
\begin{aligned}
P_{n}^{*}(A) & =\frac{1}{\phi^{n-1}} \mid A \text { and } \lambda_{\kappa_{n}}=1\left|+\frac{1}{\phi^{n-2}}\right| A \text { and } \lambda_{\kappa_{n}} \geq 2 \mid \\
& >\frac{1}{\phi^{n-2}} \mid A \text { and } \lambda_{\kappa_{n}} \geq 2 \mid=\frac{F_{n-1}}{\phi^{n-2}} P_{n}(A)
\end{aligned}
$$

and

$$
P_{n}(A) \leq \frac{\phi^{n-2}}{F_{n-1}} P_{n}^{*}(A) \leq\left(\frac{\sqrt{5}}{\phi}+O\left(\frac{1}{\phi^{2 n}}\right)\right) \frac{C_{1}}{n^{1+\phi^{2}}} \leq \frac{C_{2}}{n^{1+\phi^{2}}}
$$

\section{$5 \quad$ Asymptotic normality of $\log B$}

The following lemma is proved in [18] with techniques from [9] and [17] and Lemma 3 from this manuscript. After the lemma, we will begin the proof of Theorem 1 . In this section, we use $\Phi(x)$ to denote the standard normal distribution.

Lemma 4. For all $x$,

$$
P_{n}^{*}\left(\frac{\log \mathbf{B}-\frac{n \mu}{1+\phi^{2}}}{\sqrt{\frac{n \gamma^{2}}{1+\phi^{2}}}} \leq x\right)=\frac{1}{\sqrt{2 \pi}} \int_{-\infty}^{x} e^{-t^{2} / 2} d t+O\left(\left(\frac{\log n}{\sqrt{n}}\right)^{1 / 3}+\frac{x \log n}{\sqrt{n}}\right) .
$$

Proof of Theorem 1. For any integer $t \geq 1$,

$$
\begin{aligned}
P_{n}^{*}(\mathbf{B}=t) & =P_{n}^{*}\left(\mathbf{B}=t \text { and } \lambda_{\kappa_{n}} \geq 2\right)+P_{n}^{*}\left(\mathbf{B}=t \text { and } \lambda_{\kappa_{n}}=1\right) \\
& =\frac{1}{\phi^{n-2}} \mid \mathbf{B}_{n}=t \text { and } \lambda_{\kappa_{n}} \geq 2\left|+\frac{1}{\phi^{n-1}}\right| \mathbf{B}_{n}=t \text { and } \lambda_{\kappa_{n}}=1 \mid \\
& =\frac{1}{\phi^{n-2}} \mid \mathbf{B}_{n}=t \text { and all } \lambda_{i} \geq 2\left|+\frac{1}{\phi^{n-1}}\right| \mathbf{B}_{n-1}=t \text { and all } \lambda_{i} \geq 2 \mid \\
& =\frac{F_{n-1}}{\phi^{n-2}} P_{n}(\mathbf{B}=t)+\frac{F_{n-2}}{\phi^{n-1}} P_{n-1}(\mathbf{B}=t)
\end{aligned}
$$


where the step from (7) to (8) is made by noting the bijection between the set of compositions of $n-1$ and the set of compositions of $n$ with last part 1 . Summing over $t$ on both sides and letting $\xi_{n}=\sqrt{\frac{n \gamma^{2}}{1+\phi^{2}}} x+\frac{n \mu}{1+\phi^{2}}$, we have

$$
P_{n}^{*}\left(\log \mathbf{B} \leq \xi_{n}\right)=\frac{F_{n-2}}{\phi^{n-1}} P_{n-1}\left(\log \mathbf{B} \leq \xi_{n}\right)+\frac{F_{n-1}}{\phi^{n-2}} P_{n}\left(\log \mathbf{B} \leq \xi_{n}\right) .
$$

We would like to rewrite $P_{n-1}\left(\log \mathbf{B} \leq \xi_{n}\right)$ in terms of $P_{n-1}\left(\log \mathbf{B} \leq \xi_{n-1}\right)$, so we let $s_{n, x}=\sqrt{1+\frac{1}{n-1}}\left(x+\frac{\mu / \gamma}{\sqrt{1+\phi^{2}} \sqrt{n}}\right)$ and make the following calculation:

$$
\begin{aligned}
P_{n-1}\left(\log \mathbf{B} \leq \xi_{n}\right) & =P_{n-1}\left(\log \mathbf{B} \leq \frac{n \mu}{1+\phi^{2}}+\sqrt{\frac{n \gamma^{2}}{1+\phi^{2}}} x\right) \\
& =P_{n-1}\left(\frac{\log \mathbf{B}-\frac{(n-1) \mu}{1+\phi^{2}}}{\sqrt{\frac{(n-1) \gamma^{2}}{1+\phi^{2}}}} \leq s_{n, x}\right) \\
& =P_{n-1}\left(\log \mathbf{B} \leq \xi_{n-1}\right)+P_{n-1}\left(x<\frac{\log \mathbf{B}-\frac{(n-1) \mu}{1+\phi^{2}}}{\sqrt{\frac{(n-1) \gamma^{2}}{1+\phi^{2}}}} \leq s_{n, x}\right) .
\end{aligned}
$$

As a lower bound, we can drop the second term to get

$$
P_{n-1}\left(\log \mathbf{B} \leq \xi_{n}\right) \geq P_{n-1}\left(\log \mathbf{B} \leq \xi_{n-1}\right) .
$$

As an upper bound, we derive from (9) the inequality

$$
P_{n}^{*}(\mathbf{B}=t) \geq \frac{F_{n-1}}{\phi^{n-2}} P_{n}(\mathbf{B}=t)
$$

so that

$$
P_{n}(\mathbf{B}=t) \leq \frac{\phi^{n-2}}{F_{n-1}} P_{n}^{*}(\mathbf{B}=t)=\left(\frac{\sqrt{5}}{\phi}+O\left(\frac{1}{\phi^{2 n}}\right)\right) P_{n}^{*}(\mathbf{B}=t) .
$$

Then we can obtain an upper bound as follows, with the use of Lemma 4:

$$
\begin{aligned}
P_{n-1}\left(x<\frac{\log \mathbf{B}-\frac{(n-1) \mu}{1+\phi^{2}}}{\sqrt{\frac{(n-1) \gamma^{2}}{1+\phi^{2}}}} \leq s_{n, x}\right) & \leq \frac{\sqrt{5}}{\phi} P_{n-1}^{*}\left(x<\frac{\log \mathbf{B}-\frac{(n-1) \mu}{1+\phi^{2}}}{\sqrt{\frac{(n-1) \gamma^{2}}{1+\phi^{2}}}} \leq s_{n, x}\right)+O\left(\frac{1}{\phi^{2 n}}\right) \\
& =\frac{\sqrt{5}}{\phi}\left(\Phi\left(s_{n, x}\right)-\Phi(x)\right)+O\left(\left(\frac{\log n}{\sqrt{n}}\right)^{1 / 3}+\frac{x \log n}{\sqrt{n}}\right) \\
& =\frac{\sqrt{5}}{\phi}\left(\frac{1}{\sqrt{2 \pi}} \int_{x}^{s_{n, x}} e^{-t^{2} / 2} d t\right)+O\left(\left(\frac{\log n}{\sqrt{n}}\right)^{1 / 3}+\frac{x \log n}{\sqrt{n}}\right) \\
& \leq \frac{\sqrt{5}}{\phi \sqrt{2 \pi}}\left(s_{n, x}-x\right)+O\left(\left(\frac{\log n}{\sqrt{n}}\right)^{1 / 3}+\frac{x \log n}{\sqrt{n}}\right) .
\end{aligned}
$$


With use of the Taylor expansion $\sqrt{1+t}=1+\frac{t}{2}+O\left(t^{2}\right)$, we can show that

$$
s_{n, x}-x=O\left(\frac{1}{\sqrt{n}}+\frac{x}{n}\right) .
$$

Therefore,

$$
P_{n-1}\left(\log \mathbf{B} \leq \xi_{n}\right) \leq P_{n-1}\left(\log \mathbf{B} \leq \xi_{n-1}\right)+O\left(\left(\frac{\log n}{\sqrt{n}}\right)^{1 / 3}+\frac{x \log n}{\sqrt{n}}\right) .
$$

Going back to equation (10), we have

$$
\begin{gathered}
P_{n}^{*}\left(\log \mathbf{B} \leq \xi_{n}\right)=\frac{F_{n-2}}{\phi^{n-1}} P_{n-1}\left(\log \mathbf{B} \leq \xi_{n-1}\right)+\frac{F_{n-1}}{\phi^{n-2}} P_{n}\left(\log \mathbf{B} \leq \xi_{n}\right) \\
+O\left(\left(\frac{\log n}{\sqrt{n}}\right)^{1 / 3}+\frac{x \log n}{\sqrt{n}}\right)
\end{gathered}
$$

Letting $a_{n}=\frac{F_{n-1}}{\phi^{n}} P_{n}\left(\log \mathbf{B} \leq \xi_{n}\right)$ and $b_{n}=P_{n}^{*}\left(\log \mathbf{B} \leq \xi_{n}\right)$, we rewrite this as

$$
b_{n}=\phi^{2} a_{n}+a_{n-1}+O\left(\left(\frac{\log n}{\sqrt{n}}\right)^{1 / 3}+\frac{x \log n}{\sqrt{n}}\right) .
$$

Solving for $a_{n}$ gives

$$
a_{n}=\frac{1}{\phi^{2}} b_{n}-\frac{1}{\phi^{2}} a_{n-1}+O\left(\left(\frac{\log n}{\sqrt{n}}\right)^{1 / 3}+\frac{x \log n}{\sqrt{n}}\right) .
$$

Iterating $\lfloor n / 2\rfloor$ times, we obtain

$$
\begin{aligned}
a_{n}=\frac{1}{\phi^{2}} & \sum_{k=\lceil n / 2\rceil}^{n}\left(\frac{-1}{\phi^{2}}\right)^{n-k} b_{k}+\left(\frac{-1}{\phi^{2}}\right)^{\lfloor n / 2\rfloor+1} a_{\lceil n / 2\rceil-1} \\
& +\sum_{k=\lceil n / 2\rceil}^{n}\left(\frac{-1}{\phi^{2}}\right)^{n-k+1} \cdot O\left(\left(\frac{\log k}{\sqrt{k}}\right)^{1 / 3}+\frac{x \log k}{\sqrt{k}}\right) \\
=T_{1} & +T_{2}+T_{3} .
\end{aligned}
$$

We now look at each term separately:

$$
\begin{aligned}
\left|T_{2}\right| & =\left|\left(\frac{-1}{\phi^{2}}\right)^{\lfloor n / 2\rfloor+1} a_{\lceil n / 2\rceil-1}\right| \\
& \leq \mid\left(\frac{-1}{\phi^{2}}\right)^{\lfloor n / 2\rfloor+1} \cdot \frac{F_{\lfloor n / 2\rfloor-2}}{\phi^{\lfloor n / 2\rfloor-1} \cdot 1 \mid} \\
& =O\left(\frac{1}{\phi^{n}}\right)
\end{aligned}
$$




$$
\begin{aligned}
\left|T_{3}\right| & =\left|\sum_{k=\lceil n / 2\rceil}^{n}\left(\frac{-1}{\phi^{2}}\right)^{n-k+1} \cdot O\left(\left(\frac{\log k}{\sqrt{k}}\right)^{1 / 3}+\frac{x \log k}{\sqrt{k}}\right)\right| \\
& \leq\left|C \cdot\left(\left(\frac{\log n}{\sqrt{\lceil n / 2\rceil}}\right)^{1 / 3}+\frac{x \log n}{\sqrt{\lceil n / 2\rceil}}\right) \sum_{k=\lceil n / 2\rceil}^{n}\left(\frac{-1}{\phi^{2}}\right)^{n-k}\right| \\
& =O\left(\left(\frac{\log n}{\sqrt{n}}\right)^{1 / 3}+\frac{x \log n}{\sqrt{n}}\right) .
\end{aligned}
$$

With use of Lemma 4, we have

$$
\begin{aligned}
T_{1} & =\frac{1}{\phi^{2}} \sum_{k=\lceil n / 2\rceil}^{n}\left(\frac{-1}{\phi^{2}}\right)^{n-k} \Phi(x)+\frac{1}{\phi^{2}} \sum_{k=\lceil n / 2\rceil}^{n}\left(\frac{-1}{\phi^{2}}\right)^{n-k} \cdot O\left(\left(\frac{\log k}{\sqrt{k}}\right)^{1 / 3}+\frac{x \log k}{\sqrt{k}}\right) \\
& =T_{1, a}+T_{1, b} .
\end{aligned}
$$

We again look at each term separately:

$$
\begin{gathered}
T_{1, b}=O\left(T_{3}\right) \\
T_{1, a}=\frac{1}{\phi^{2}} \sum_{k=\lceil n / 2\rceil}^{n}\left(\frac{-1}{\phi^{2}}\right)^{n-k} \Phi(x) \\
=\Phi(x) \frac{1}{\phi^{2}} \sum_{k=0}^{\lfloor n / 2\rfloor}\left(\frac{-1}{\phi^{2}}\right)^{k} \\
=\frac{\Phi(x)}{1+\phi^{2}}+O\left(\frac{1}{\phi^{n}}\right) .
\end{gathered}
$$

Putting the estimates from (11), (12), (13), and (14) together, we get

$$
a_{n}=\frac{\Phi(x)}{1+\phi^{2}}+O\left(\left(\frac{\log n}{\sqrt{n}}\right)^{1 / 3}+\frac{x \log n}{\sqrt{n}}\right) .
$$

Recalling that $a_{n}=\frac{F_{n-1}}{\phi^{n}} P_{n}\left(\log \mathbf{B} \leq \xi_{n}\right)$, we therefore have

$$
P_{n}\left(\log \mathbf{B} \leq \xi_{n}\right)=\frac{\phi^{n}}{F_{n-1}} a_{n}=\left(\phi \sqrt{5}+O\left(\frac{1}{\phi^{2 n}}\right)\right) a_{n} .
$$

Finally,

$$
\begin{aligned}
P_{n}\left(\log \mathbf{B} \leq \xi_{n}\right) & =\Phi(x) \frac{\phi \sqrt{5}}{1+\phi^{2}}+O\left(\left(\frac{\log n}{\sqrt{n}}\right)^{1 / 3}+\frac{x \log n}{\sqrt{n}}\right) \\
& =\Phi(x)+O\left(\left(\frac{\log n}{\sqrt{n}}\right)^{1 / 3}+\frac{x \log n}{\sqrt{n}}\right) .
\end{aligned}
$$


Therefore the rate of convergence is uniform as long as $\frac{|x| \log n}{\sqrt{n}} \leq\left(\frac{\log n}{\sqrt{n}}\right)^{1 / 3}$, i.e. as $\operatorname{long}$ as $|x| \leq\left(\frac{\sqrt{n}}{\log n}\right)^{2 / 3}$

\section{References}

[1] Cyril Banderier and Paweł Hitczenko. Enumeration and asymptotics of restricted compositions having the same number of parts. Preprint available at http://www.math.drexel.edu/ phitczen/BaHi5.pdf, 2011.

[2] Edward A. Bender and E. Rodney Canfield. Locally restricted compositions I. Restricted adjacent differences. Electron. J. Combin., 12:R57, 2005.

[3] Edward A. Bender and E. Rodney Canfield. Locally restricted compositions II. General restrictions and infinite matrices. Electron. J. Combin., 16(1):R108, 2009.

[4] Edward A. Bender and E. Rodney Canfield. Locally restricted compositions III. Adjacent-part periodic inequalities. Electron. J. Combin., 17(1):R145, 2010.

[5] Miklós Bóna and Arnold Knopfmacher. On the probability that certain compositions have the same number of parts. Ann. Comb., 14(3):291-306, 2010.

[6] Phyllis Chinn and Silvia Heubach. Integer sequences related to compositions without 2's. J. Integer Seq., 6(2):Article 03.2.3, 2003.

[7] Philippe Flajolet and Robert Sedgewick. Analytic combinatorics. Cambridge University Press, Cambridge, 2009.

[8] William M. Y. Goh and Paweł Hitczenko. Average number of distinct part sizes in a random Carlitz composition. European J. Combin., 23(6):647-657, 2002.

[9] Allan Gut and Svante Janson. The limiting behaviour of certain stopped sums and some applications. Scand. J. Statist., 10(4):281-292, 1983.

[10] Paweł Hitczenko and Arnold Knopfmacher. Gap-free compositions and gap-free samples of geometric random variables. Discrete Math., 294(3):225-239, 2005.

[11] Paweł Hitczenko and Guy Louchard. Distinctness of compositions of an integer: a probabilistic analysis. Random Structures Algorithms, 19(3-4):407-437, 2001. Analysis of algorithms (Krynica Morska, 2000).

[12] Paweł Hitczenko and Carla D. Savage. On the multiplicity of parts in a random composition of a large integer. SIAM J. Discrete Math., 18(2):418-435, 2004.

[13] Boris L. Kheyfets. The number of part sizes of a given multiplicity in a random Carlitz composition. Adv. in Appl. Math., 35(3):335-354, 2005.

[14] A. Knopfmacher and B. Richmond. Compositions with distinct parts. Aequationes Math., 49(1-2):86-97, 1995.

[15] Arnold Knopfmacher and Helmut Prodinger. On Carlitz compositions. European J. Combin., 19(5):579-589, 1998. 
[16] Arnold Knopfmacher and Neville Robbins. Compositions with parts constrained by the leading summand. Ars Combin., 76:287-295, 2005.

[17] A. Rényi. On the asymptotic distribution of the sum of a random number of independent random variables. Acta Math. Acad. Sci. Hungar., 8:193-199, 1957.

[18] Caroline Shapcott. Dissertation (in preparation). PhD thesis, Drexel University. 